\title{
Pulmonary adenocarcinoma with high-grade fetal adenocarcinoma component has a poor prognosis, comparable to that of micropapillary adenocarcinoma
}

\author{
Masaki Suzuki ${ }^{1}$ Yukio Nakatani ${ }^{2} \cdot$ Hiroyuki Ito $^{3} \cdot$ Hiroto Narimatsu $^{4} \cdot \mathrm{Kozo}$ Yamada $^{5} \cdot$ Emi Yoshioka $^{1} \cdot$

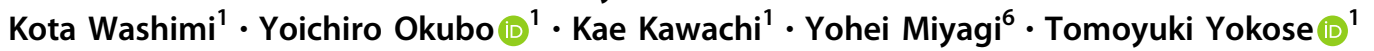

Received: 14 December 2017 / Revised: 9 March 2018 / Accepted: 11 March 2018 / Published online: 21 May 2018

(c) United States \& Canadian Academy of Pathology 2018

\begin{abstract}
Fetal adenocarcinoma is a rare variant of lung adenocarcinoma, which is subcategorized into low-grade and high-grade forms. High-grade fetal adenocarcinoma confers worse prognosis than low-grade fetal adenocarcinoma, but the prognostic differences between high-grade fetal adenocarcinoma and conventional lung adenocarcinoma are unknown. We reviewed tissue sections of 3719 cases of surgically resected primary lung cancers and found 53 lung cancers with a high-grade fetal adenocarcinoma component. We analyzed their clinicopathological and immunohistochemical features, and performed a prognostic analysis of adenocarcinomas with the fetal-type component. We further analyzed the prognostic differences between adenocarcinomas with the fetal-type component and conventional adenocarcinomas without the fetal-type component. Lung cancers with the fetal-type component predominantly occurred in elderly men with a smoking history. Twenty-nine patients had stage I disease, 13 patients had stage II, and 11 patients had stage III. The fetal-type histology was combined with conventional-type adenocarcinoma (41 cases), squamous cell carcinoma (5 cases), large cell neuroendocrine carcinoma (5 cases), enteric adenocarcinoma ( 2 cases), and small cell carcinoma ( 1 case). The fetal-type component showed immunopositivity for $\alpha$-fetoprotein (39\%), glypican-3 (37\%), and SALL4 (17\%). The 5-year overall survivals of fetal-typepredominant and fetal-type-nonpredominant patients were 44 and $56 \%$, respectively $(P=0.962)$. The 5 -year overall survivals of lepidic-, acinar-, papillary-, solid-, and micropapillary-predominant adenocarcinomas, invasive mucinous adenocarcinomas, and adenocarcinomas with the fetal-type component were 94, 82, 77, 69, 57, 83, and 41\%, respectively $(P$ $<0.001)$. Univariate and multivariate analyses showed that adenocarcinomas with the fetal-type component had a significantly lower overall survival rate than the other histological subtypes, except for the micropapillary-predominant subtype. Our study demonstrated that adenocarcinomas with the fetal-type component had a poor prognosis that was comparable to that of micropapillary adenocarcinoma. The presence of the high-grade fetal adenocarcinoma component in lung adenocarcinomas is an important prognostic marker.
\end{abstract}

Electronic supplementary material The online version of this article (https://doi.org/10.1038/s41379-018-0057-z) contains supplementary material, which is available to authorized users.

Masaki Suzuki

m-suzuki@kcch.jp

1 Department of Pathology, Kanagawa Cancer Center, Yokohama, Kanagawa, Japan

2 Department of Diagnostic Pathology, Graduate School of Medicine, Chiba University, Chiba, Chiba, Japan

3 Department of Thoracic Surgery, Kanagawa Cancer Center,

\section{Introduction}

Fetal adenocarcinoma of the lung is a rare variant of lung adenocarcinoma, which is subcategorized into low-grade and high-grade forms [1, 2]. Although both forms are characterized by complex glandular structures resembling

\section{Yokohama, Kanagawa, Japan}

4 Cancer Prevention and Control Division, Kanagawa Cancer Center Research Institute, Yokohama, Kanagawa, Japan

5 Department of Thoracic Oncology, Kanagawa Cancer Center, Yokohama, Kanagawa, Japan

6 Molecular Pathology and Genetics Division, Kanagawa Cancer Center Research Institute, Yokohama, Kanagawa, Japan 
Table 1 Antibodies used for the immunohistochemical studies

\begin{tabular}{lllll}
\hline Antibody & Clone & Type & Source & Dilution \\
\hline$\alpha$-fetoprotein & - & Rabbit, polyclonal & Nichirei, Tokyo, Japan & Ready-to-use \\
Glypican-3 & $1 \mathrm{G} 12$ & Mouse, monoclonal & BioMosaics, Burlington, VT, USA & $1: 100$ \\
SALL4 & $6 \mathrm{E} 3$ & Mouse, monoclonal & Sigma-Aldrich, Saint Louis, MO, USA & $1: 2000$ \\
TTF-1 & $8 \mathrm{G} 7 \mathrm{G} 3 / 1$ & Mouse, monoclonal & Dako, Glostrup, Denmark & $1: 200$ \\
$\beta$-catenin & 14/Beta-Catenin & Mouse, monoclonal & BD Biosciences, Franklin Lakes, NJ, USA & $1: 200$ \\
CDX2 & AMT28 & Mouse, monoclonal & Santa Cruz Biotechnology, Dallas, TX, USA & $1: 50$ \\
Chromogranin A & DAK-A3 & Mouse, monoclonal & Dako, Glostrup, Denmark & $1: 200$ \\
Synaptophysin & $27 G 12$ & Mouse, monoclonal & Leica, Newcastle Upon Tyne, UK & $1: 200$ \\
CD56 (NCAM) & 1B6 & Mouse, monoclonal & Nichirei, Tokyo, Japan & Ready-to-use \\
MUC2 & Ccp58 & Mouse, monoclonal & Leica, Newcastle Upon Tyne, UK & $1: 100$ \\
Cytokeratin 7 & OV-TL 12/30 & Mouse, monoclonal & Dako, Glostrup, Denmark & $1: 50$ \\
Cytokeratin 20 & Ks20.8 & Mouse, monoclonal & Dako, Glostrup, Denmark & Ready-to-use \\
p40 & - & Rabbit, polyclonal & Nichirei, Tokyo, Japan & \\
\hline
\end{tabular}

airway epithelium in the pseudoglandular phase of the fetal lung, they have different clinicopathological features. Lowgrade fetal adenocarcinoma commonly appears in young- to middle-aged women with a smoking history, and is usually diagnosed during stage I [1, 3, 4]. Low-grade fetal adenocarcinoma histologically displays mild nuclear atypia and a characteristic morula formation [1, 3, 4]. On the other hand, high-grade fetal adenocarcinoma predominantly occurs in elderly men with a heavy smoking history, and is often diagnosed at advanced stages [1, 4-6]. High-grade fetal adenocarcinoma typically shows prominent nuclear atypia and no morula formation [1, 4-6]. In addition, high-grade fetal adenocarcinoma frequently coexists with a conventional adenocarcinoma component, and is occasionally combined with other carcinoma components, such as large cell neuroendocrine carcinoma and enteric adenocarcinoma [4-6]. It is known that high-grade fetal adenocarcinoma confers worse prognosis than low-grade fetal adenocarcinoma, but the prognostic differences between high-grade fetal adenocarcinoma and the major subtypes of lung adenocarcinoma have yet to be clarified.

The aim of the present study is to clarify the clinicopathological, immunohistochemical, and prognostic features of high-grade fetal adenocarcinoma. We collected 53 cases of lung cancer with a high-grade fetal adenocarcinoma component in various proportions. To examine the prognostic contribution of high-grade fetal adenocarcinoma, we compared and analyzed the prognoses of lung adenocarcinomas with the fetal-type component and conventional adenocarcinomas without the fetal-type component. Additionally, we investigated whether a nonpredominant fetaltype component influenced the prognosis of adenocarcinomas with the fetal-type component. To our knowledge, this is the first study describing a detailed prognostic analysis of high-grade fetal adenocarcinoma.

\section{Materials and methods}

\section{Patients}

The ethics committee of the Kanagawa Cancer Center approved this study. We reviewed tissue sections of 3719 cases of surgically resected primary lung cancer from the Kanagawa Cancer Center from January 1991 to December 2016, and found 53 lung cancer cases with a high-grade fetal adenocarcinoma component in varying proportions of at least $5 \%$ of the total tumor volume. A glandular pattern of the fetal-type component was defined as follows: (1) the complex glandular, cribriform, and papillary structures were composed of clear columnar cells with pseudostratified nuclei and prominent nuclear atypia; (2) the apical border of the tumoral glands was typically flat; and (3) no morula formation was observed. Additionally, we defined a solidclear cell pattern of the fetal-type component as solid growth of tumor cells with clear cytoplasm that showed a transition from a glandular pattern of the fetal-type component. Glass slides from all cases were reviewed by two or more pulmonary pathologists (MS, YN, and TY).

We performed prognostic analysis of adenocarcinomas with the fetal-type component (41 cases, 1991-2016), after having excluded the cases that harbored nonadenocarcinomatous components and the cases that underwent limited resection. Additionally, to evaluate the prognostic contribution of high-grade fetal adenocarcinoma, we compared and analyzed the prognostic differences between adenocarcinomas with the fetal-type component (36 cases, 2000-2016) and major subtypes of adenocarcinoma without any fetal-type component (1514 cases, 2000-2016). The major subtype cases were extracted from the Thoracic Surgical Database of Kanagawa Cancer Center, and were classified into the following 
six histological subgroups, according to the International Association for the Study of Lung Cancer/American Thoracic Society/European Respiratory Society adenocarcinoma classification: (1) lepidic-predominant, (2) acinar-predominant, (3) papillary-predominant, (4) solidpredominant, (5) micropapillary-predominant, and (6) invasive mucinous adenocarcinomas [7]. In this study, cases of adenocarcinoma in situ and minimally invasive adenocarcinoma were excluded from the lepidicpredominant subgroup.

The clinical data were retrieved from the Thoracic Surgical Database (after 2000) and medical records (before 2000). Patients were excluded from this study if they underwent chemotherapy or radiotherapy before surgery, underwent incomplete resection, or had incomplete followup data. Although the Union for International Cancer Control (UICC) published the 8th edition of the UICC TNM classification of malignant tumors in December 2016 [8], this study is retrospective, and it was difficult to measure accurate invasive sizes of old lung cancer cases. Therefore, lung cancer stages were evaluated in accordance with the 7th edition of the UICC TNM classification in this study [9].

\section{Histologic evaluation}

All resected specimens were fixed in $10 \%$ formalin, embedded in paraffin, and stained with hematoxylin and eosin. As necessary, periodic acid-Schiff staining was performed to evaluate cytoplasmic glycogen. The amount of the fetal-type component was evaluated as a percentage of the total tumor volume in hematoxylin and eosin sections. Other components were also assessed and classified according to the World Health Organization (WHO) classification of lung tumors [2]. Pleural invasion, lymphovascular invasion, and lymph node metastasis were also assessed.

\section{Immunohistochemistry}

We selected a representative slide of each case and performed immunohistochemical analysis with primary antibodies as listed in Table 1 . We conducted immunostaining for chromogranin A, synaptophysin, CD56 (NCAM), CDX2, MUC2, cytokeratin 7, cytokeratin 20, and p40 as needed. Antigen retrieval was performed by heating slides in a PT Link module (Dako, Glostrup, Denmark). Staining was performed using an automated immunostainer (HISTOSTAINER48A; Nichirei, Tokyo, Japan), and this was followed by antibody detection with the EnVision FLEX visualization system (Dako). Appropriate positive and negative control tissues were used. Immunostaining for $\alpha$ fetoprotein was considered positive if staining was found in
Table 2 Clinicopathological features of lung cancers with high-grade fetal adenocarcinoma component

\begin{tabular}{|c|c|}
\hline Total number $(n)$ & 53 \\
\hline \multicolumn{2}{|l|}{ Age (year) } \\
\hline Median & 67 \\
\hline Range & $47-87$ \\
\hline \multicolumn{2}{|l|}{$\operatorname{Sex}(n)$} \\
\hline Female & 10 \\
\hline Male & 43 \\
\hline \multicolumn{2}{|l|}{ Smoking $(n)$} \\
\hline Never & 4 \\
\hline Ever & 41 \\
\hline No data & 8 \\
\hline \multicolumn{2}{|l|}{ Tumor size (mm) } \\
\hline Median & 33 \\
\hline Range & $18-122$ \\
\hline \multicolumn{2}{|l|}{ Pleural invasion $(n)$} \\
\hline Absent & 27 \\
\hline Present & 26 \\
\hline \multicolumn{2}{|l|}{ Lymphovascular invasion $(n)$} \\
\hline Absent & 38 \\
\hline Present & 15 \\
\hline \multicolumn{2}{|l|}{ Stage $(n)$} \\
\hline I & 29 \\
\hline II & 13 \\
\hline III & 11 \\
\hline \multicolumn{2}{|l|}{ Mixed components $(n)$} \\
\hline Conventional adenocarcinoma & 41 \\
\hline Squamous carcinoma & 5 \\
\hline Large cell neuroendocrine carcinoma & 5 \\
\hline Invasive mucinous adenocarcinoma & 5 \\
\hline Enteric adenocarcinoma & 2 \\
\hline Small cell carcinoma & 1 \\
\hline Sarcomatoid component & 3 \\
\hline
\end{tabular}

$>1 \%$ of the tumor cells. In other immunostaining, staining of $>10 \%$ of tumor cells was considered to indicate positivity.

\section{Statistical analysis}

Statistical comparisons were performed using the Fisher's test. Five-year overall survival and disease-specific survival rates were calculated using the Kaplan-Meier method, and prognostic factors were evaluated using the log-rank test or Cox model. In all statistical tests, $P$ values $<0.05$ were considered statistically significant. Statistical analysis was conducted using EZR version 1.36 (Saitama Medical Center, Jichi Medical University, Saitama, Japan), a graphical user interface for R (The R Foundation for Statistical Computing, Vienna, Austria) [10]. 

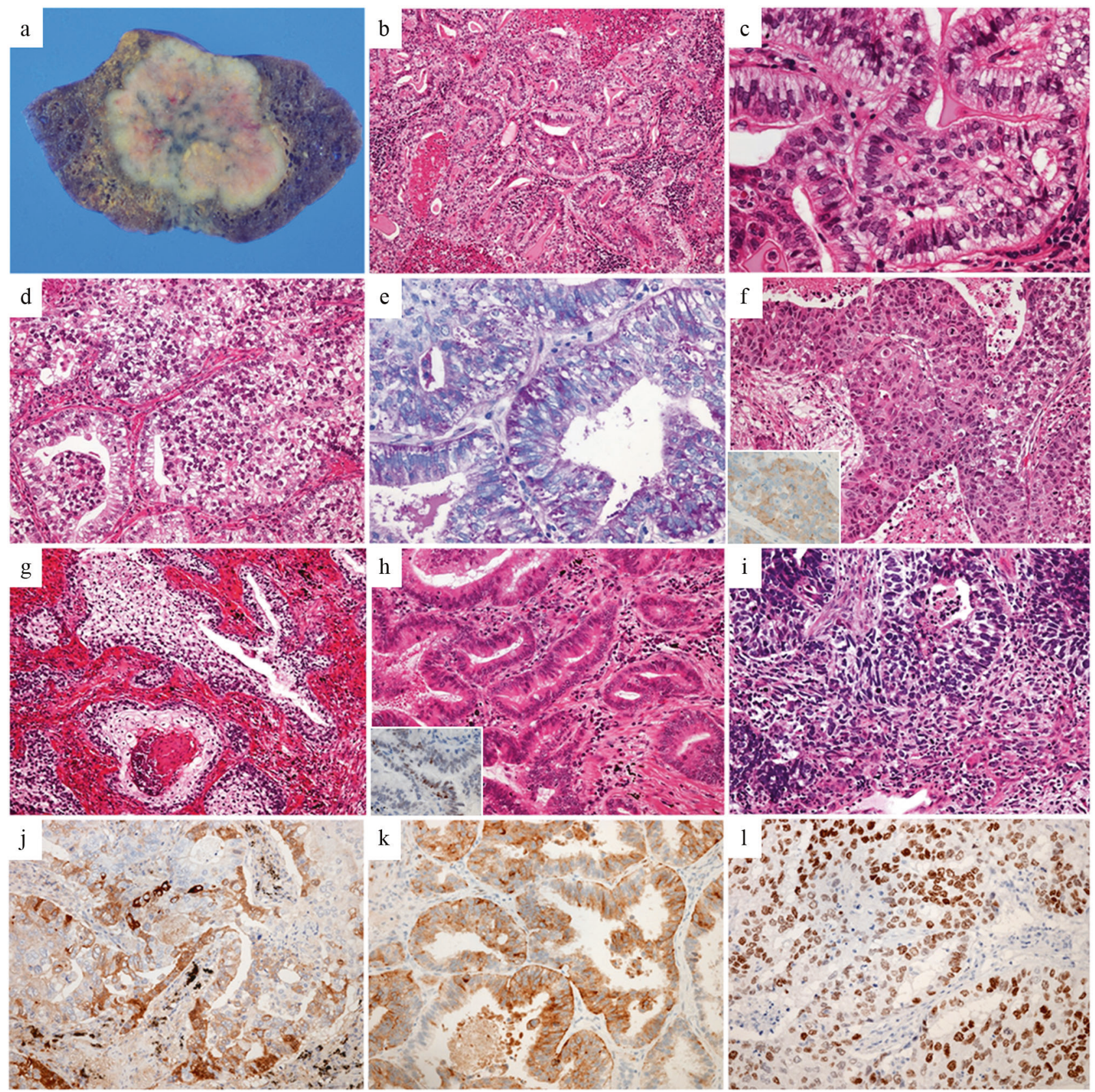

Fig. 1 Macroscopic, microscopic, and immunohistochemical characteristics of lung adenocarcinoma with a high-grade fetal adenocarcinoma component. The cut surface was a lobulated, gray-white, solid tumor with focal necrosis (a). The fetal-type component exhibited complex glandular structures (b) composed of columnar cells with clear cytoplasm and pseudostratified nuclei (c). Coexistence of a glandular pattern and a solid-clear cell pattern were often seen $(\mathbf{d})$. Periodic acid-Schiff stain showed glycogen-rich cytoplasm in the tumor cells of the fetal-type component (e). Highgrade fetal adenocarcinoma combined with large cell neuroendocrine carcinoma (f: inset shows tumor cells with immunoexpression of synaptophysin), squamous cell carcinoma (g), enteric adenocarcinoma (h: inset shows tumor cells with immunoexpression of CDX2), and sarcomatoid components (i). The fetal-type component occasionally displayed immunoexpression of $\alpha$-fetoprotein (j), glypican-3 (k), and SALL4 (l)

\section{Results}

\section{Clinicopathologic features}

The clinicopathological findings of the 53 cases of lung cancer with the fetal-type component are summarized in
Table 2, and the pathological characteristics of the fetal-type component are shown in Fig. 1. Lung cancers with the fetaltype component predominantly occurred in elderly men with a smoking history. Pulmonary lobectomy was performed in 52 cases and partial resection in 1 case. The median tumor size was $33 \mathrm{~mm}$. Twenty-nine patients had 

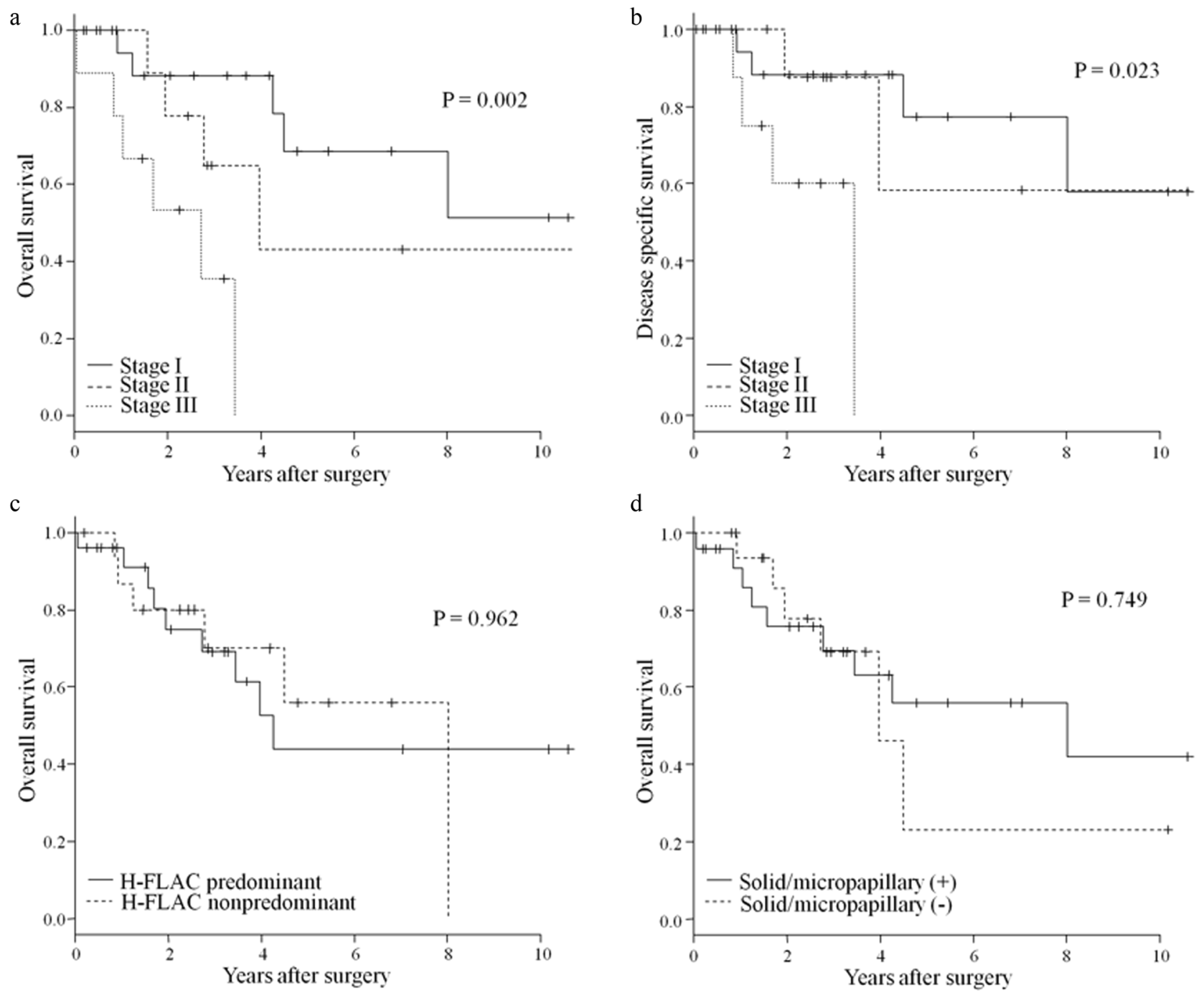

Fig. 2 Kaplan-Meier survival curves of patients with lung adenocarcinomas with a high-grade fetal adenocarcinoma component. Overall survival (a) and disease-specific survival (b) curves of adenocarcinomas with the fetal-type component for each stage. Overall

Table 3 Summary of immunohistochemical analysis

\begin{tabular}{llll}
\hline & \multicolumn{2}{l}{$\begin{array}{l}\text { High-grade fetal } \\
\text { adenocarcinoma }(\%)\end{array}$} & \\
\cline { 2 - 3 } & Glandular & Solid-clear & \\
\hline a-fetoprotein & $20 / 52(39)$ & $6 / 15(40)$ & $P=1$ \\
Glypican-3 & $19 / 52(37)$ & $7 / 15(47)$ & $P=0.553$ \\
SALL4 & $9 / 52(17)$ & $4 / 15(27)$ & $P=0.466$ \\
TTF-1 & $33 / 52(64)$ & $10 / 15(67)$ & $P=1$ \\
\hline
\end{tabular}

stage I disease, 13 patients had stage II, and 11 patients had stage III. Macroscopically, the cut surfaces of most cases showed lobulated, gray-white, solid tumors with focal necrosis. Lung cancers with the fetal-type component showed the fetal-type histology in various proportions

survival curves of adenocarcinomas with the fetal-type component separated by the proportion of fetal-type component (c) and presence/ absence of solid and/or micropapillary components (d)

(5-9\% in 7 cases, $10-19 \%$ in $5,20-29 \%$ in $4,30-39 \%$ in 3 , $40-49 \%$ in $3,50-59 \%$ in $1,60-69 \%$ in $5,70-79 \%$ in 7 , $80-89 \%$ in $6,90-99 \%$ in 7 , and $100 \%$ in 5). Five cases consisted of the fetal-type histology in a pure form, and the other cases showed the fetal-type histology combined with diverse components (conventional adenocarcinoma in 30 cases, invasive mucinous adenocarcinoma in 1, conventional adenocarcinoma and invasive mucinous adenocarcinoma in 4, conventional adenocarcinoma and enteric adenocarcinoma in 2, large cell neuroendocrine carcinoma in 4, conventional adenocarcinoma and large cell neuroendocrine carcinoma in 1 , conventional adenocarcinoma and squamous cell carcinoma in 2, small cell carcinoma and squamous cell carcinoma in 1 , minor sarcomatoid component in 1, and conventional adenocarcinoma, squamous cell carcinoma, and minor sarcomatoid component in 2). The 
coexisting components of conventional adenocarcinoma showed various patterns, including lepidic (22 cases), acinar (19 cases), papillary (27 cases), solid (3 cases), and micropapillary (15 cases) patterns. Among 16 cases of adenocarcinoma with a non-predominant fetal-type component (the fetal-type component $<50 \%$ of the tumor), there were $1,7,6,1$, and 1 cases of lepidic-, acinar-, papillary-, and solid-predominant adenocarcinoma, and mixed invasive mucinous and non-mucinous adenocarcinoma, respectively. Sarcomatoid histology was recognized as a minor component in 3 cases $(<5 \%$ of the total tumor volume in 2 and $15 \%$ in 1). A solid-clear cell pattern of the fetal-type component was observed in 14 cases. Among the 53 cases of lung cancer with the fetal-type component, 27 satisfied the diagnostic criteria of high-grade fetal adenocarcinoma in the WHO classification, which defines high-grade fetal adenocarcinoma as a tumor that predominantly contains this component, and it accounted for $0.73 \%$ of primary lung cancer cases at our institution. Tumor cells of the fetal-type component had glycogen granules, which were stained by periodic acid-Schiff stain in all cases. Severe emphysematous changes and pulmonary fibrosis of usual interstitial pneumonia pattern were observed in the background lung tissue in 10 and 6 cases, respectively.

\section{Immunohistochemical features}

The immunohistochemical findings are shown in Table 3 and Fig. 1. Both glandular and solid-clear cell patterns of the fetal-type component occasionally showed immunopositivity for TTF-1 and oncofetal markers such as $\alpha$-fetoprotein, glypican-3, and SALL4. There were no significant differences in the expression of any marker between the two patterns of the fetal-type component. At least one of the three oncofetal markers was positive in $60 \%$ of the fetal-type components. $\beta$-catenin was mainly membrane-localized in all cases. The squamous cell carcinoma component was positive for $\mathrm{p} 40$. The large cell neuroendocrine carcinoma and small cell carcinoma components were positive for neuroendocrine markers such as chromogranin A, synaptophysin, and CD56. The enteric adenocarcinoma component was positive for CDX2, MUC2, or cytokeratin 20.

\section{Prognostic analysis of adenocarcinomas with the fetal-type component}

We analyzed prognosis in 41 cases of lung adenocarcinoma with the fetal-type component (1991-2016), which lacked non-adenocarcinomatous components. The median followup period was 994 days (range, 71-8505 days). The 5-year overall and disease-specific survival rates of all cases were 49 and $63 \%$, respectively. The 5-year overall survival rates in patients with stage I and II disease were 69 and $43 \%$,
Table 4 Univariate analysis for OS and DSS of adenocarcinomas with H-FLAC

\begin{tabular}{|c|c|c|c|c|c|}
\hline & Number & $\begin{array}{l}5 \text {-yr OS } \\
(\%)\end{array}$ & $\begin{array}{l}P \\
\text { value }\end{array}$ & $\begin{array}{l}5 \text {-yr } \\
\text { DSS (\%) }\end{array}$ & $\begin{array}{l}P \\
\text { value }\end{array}$ \\
\hline \multicolumn{6}{|l|}{ Age (years) } \\
\hline$\leq 65$ & 17 & 47 & 0.796 & 76 & 0.360 \\
\hline$>65$ & 24 & 51 & & 56 & \\
\hline \multicolumn{6}{|l|}{ Sex } \\
\hline Female & 9 & 75 & 0.548 & 75 & 0.866 \\
\hline Male & 32 & 39 & & 56 & \\
\hline \multicolumn{6}{|l|}{ Smoking } \\
\hline Never & 4 & NA & 0.422 & NA & 0.175 \\
\hline Ever & 31 & 42 & & 61 & \\
\hline \multicolumn{6}{|l|}{ Tumor size (mm) } \\
\hline$\leq 30$ & 17 & 48 & 0.353 & 59 & 0.466 \\
\hline$>30$ & 24 & 50 & & 68 & \\
\hline \multicolumn{6}{|l|}{ Pleural invasion } \\
\hline Absent & 20 & 64 & 0.138 & 87 & 0.056 \\
\hline Present & 21 & 39 & & 46 & \\
\hline \multicolumn{6}{|l|}{ Lymphovascular invasion } \\
\hline Absent & 14 & 65 & 0.752 & 82 & 0.487 \\
\hline Present & 27 & 47 & & 60 & \\
\hline \multicolumn{6}{|l|}{ Stage } \\
\hline I & 23 & 69 & 0.002 & 77 & 0.023 \\
\hline II & 9 & 43 & & 58 & \\
\hline III & 9 & NA & & NA & \\
\hline \multicolumn{6}{|c|}{ Proportion of H-FLAC component } \\
\hline Predominant ( $\geq 50 \%)$ & 25 & 44 & 0.962 & 63 & 0.545 \\
\hline Non-predominant $(<50 \%)$ & 16 & 56 & & 64 & \\
\hline \multicolumn{6}{|l|}{ Solid/micropapillary patterns } \\
\hline Absent & 17 & 23 & 0.749 & 26 & 0.331 \\
\hline Present & 24 & 56 & & 77 & \\
\hline
\end{tabular}

OS overall survival, DSS disease-specific survival, H-FLAC highgrade fetal adenocarcinoma of the lung, $N A$ not applicable

respectively (Fig. 2a), and the 5-year disease-specific survival rates in patients with stage I and II disease were $77 \%$ and $58 \%$, respectively (Fig. 2b). The univariate analysis is summarized in Table 4. Among various clinicopathological factors, including age, sex, smoking, tumor size, pleural invasion, lymphovascular invasion, and tumor stage, only tumor stage was significantly associated with overall survival $(P=0.002)$ and disease-specific survival $(P=0.023)$. To evaluate the prognostic contribution of the proportion of the fetal-type component in the tumors, we analyzed the prognostic difference between fetal-type-predominant cases (high-grade fetal adenocarcinoma component $\geq 50 \%$ of the tumor) and fetal-type-nonpredominant cases $(<50 \%)$, and there were no significant differences in overall and diseasespecific survival between the two subgroups (Table 4 and Fig. 2c). In addition, we divided the 41 cases into two subgroups, namely, adenocarcinomas with solid and/or micropapillary patterns, including the solid-clear cell pattern, and adenocarcinomas without these patterns, and the two subgroups showed no significant differences in overall and disease-specific survival (Table 4 and Fig. 2d). In the multivariate analysis adjusted for age and tumor stage, 
Table 5 Clinicopathological features of adenocarcinomas with H-FLAC and major subtypes of lung adenocarcinoma

\begin{tabular}{|c|c|c|c|c|c|c|c|c|}
\hline & Total & $\begin{array}{l}\text { Adenocarcinomas } \\
\text { with H-FLAC }\end{array}$ & Lepidic & Acinar & Papillary & Solid & Micropapillary & $\begin{array}{l}\text { Invasive } \\
\text { mucinous }\end{array}$ \\
\hline Number & 1550 & 36 & 447 & 442 & 309 & 204 & 29 & 83 \\
\hline \multicolumn{9}{|l|}{ Age (year) } \\
\hline Median & 68 & 67 & 69 & 68 & 67 & 66 & 68 & 72 \\
\hline Range & $29-93$ & $47-87$ & $36-86$ & $35-90$ & $35-88$ & $29-82$ & $38-83$ & $32-85$ \\
\hline \multicolumn{9}{|l|}{ Sex } \\
\hline Female & 758 & 8 & 244 & 230 & 158 & 60 & 10 & 48 \\
\hline Male & 792 & 28 & 203 & 212 & 151 & 144 & 19 & 35 \\
\hline \multicolumn{9}{|l|}{ Smoking } \\
\hline Never & 705 & 5 & 249 & 214 & 137 & 47 & 10 & 43 \\
\hline Ever & 832 & 30 & 192 & 226 & 170 & 155 & 19 & 40 \\
\hline No data & 13 & 1 & 6 & 2 & 2 & 2 & 0 & 0 \\
\hline \multicolumn{9}{|c|}{ Tumor size (mm) } \\
\hline Median & 28 & 36 & 27 & 26 & 28 & 28 & 33 & 32 \\
\hline Range & $7-210$ & $17-122$ & $7-120$ & $7-160$ & $8-90$ & $8-102$ & $14-62$ & $10-210$ \\
\hline \multicolumn{9}{|c|}{ Pleural invasion } \\
\hline Absent & 1120 & 15 & 405 & 306 & 199 & 103 & 18 & 74 \\
\hline Present & 430 & 21 & 42 & 136 & 110 & 101 & 11 & 9 \\
\hline \multicolumn{9}{|c|}{ Lymphovascular invasion } \\
\hline Absent & 888 & 11 & 390 & 236 & 129 & 44 & 6 & 72 \\
\hline Present & 662 & 25 & 57 & 206 & 180 & 160 & 23 & 11 \\
\hline \multicolumn{9}{|l|}{ Stage } \\
\hline I & 1112 & 19 & 415 & 324 & 180 & 109 & 13 & 52 \\
\hline II & 245 & 9 & 23 & 60 & 72 & 49 & 5 & 27 \\
\hline III & 187 & 8 & 9 & 57 & 57 & 41 & 11 & 4 \\
\hline IV & 6 & 0 & 0 & 1 & 0 & 5 & 0 & 0 \\
\hline
\end{tabular}

H-FLAC high-grade fetal adenocarcinoma of the lung

proportion of the fetal-type component (predominant vs. non-predominant) and presence of solid/micropapillary patterns were not significant prognostic factors for overall or disease-specific survival.

\section{Prognostic analysis of adenocarcinomas with the fetal-type component and major subtypes of lung adenocarcinoma}

The clinicopathological characteristics of lung adenocarcinomas with the fetal-type component and major subtypes of lung adenocarcinoma (2000-2016) are summarized in Table 5, and the univariate analysis is shown in Table 6. The 5-year overall survival rates of lepidic-, acinar-, papillary-, solid-, and micropapillary-predominant adenocarcinomas, invasive mucinous adenocarcinomas, and adenocarcinomas with the fetal-type component were 94, 82, 77, 69, 57, 83, and $41 \%$, respectively $(P<0.001)$ (Fig. 3a). The 5-year disease-specific survival rates of lepidic-, acinar-, papillary-, solid-, and micropapillary-predominant adenocarcinomas, invasive mucinous adenocarcinomas, and adenocarcinomas with the fetal-type component were 98, 87, 85, 74, 63, 93, and $57 \%$, respectively $(P<0.001)$ (Fig. 3b). Univariate analysis showed that adenocarcinomas with the fetal-type component had significantly lower overall and diseasespecific survival rates than all the other histological subtypes, except for the micropapillary-predominant subtype (Table 6). Multivariate analysis showed that adenocarcinomas with the fetal-type component had a significantly lower overall survival rate than all the other histological subtypes, except for the micropapillary-predominant subtype, and a lower disease-specific survival rate than all the other histological subtypes, except for the micropapillary- and solidpredominant subtypes (Table 7).

In addition, we divided these subgroups into early stage (stage I) and advanced stage (stage II-IV), and performed prognostic analyses. In the early stage, the 5-year overall survival rates of lepidic-, acinar-, papillary-, solid-, and micropapillary-predominant adenocarcinomas, invasive mucinous adenocarcinomas, and adenocarcinomas with the fetal-type component were 95, 89, 87, 85, 67, 91, and 57\%, respectively $(P<0.001)$ (Fig. 3c). The 5-year disease- 
Table 6 Univariate analysis for OS and DSS of adenocarcinomas with H-FLAC and major subtypes of lung adenocarcinoma in all stage

\begin{tabular}{|c|c|c|c|c|c|}
\hline & \multicolumn{5}{|c|}{ Univariate analysis (log-rank test) } \\
\hline & Number & $\begin{array}{l}\text { 5-yr OS } \\
(\%)\end{array}$ & $P$ value & 5-yr DSS (\%) & $P$ value \\
\hline \multicolumn{6}{|l|}{ Age (year) } \\
\hline$\leq 65$ & 626 & 85 & \multirow[t]{2}{*}{$<0.001$} & 88 & \multirow[t]{2}{*}{0.264} \\
\hline$>65$ & 924 & 79 & & 87 & \\
\hline \multicolumn{6}{|l|}{ Sex } \\
\hline Female & 758 & 86 & \multirow[t]{2}{*}{$<0.001$} & 91 & \multirow[t]{2}{*}{0.002} \\
\hline Male & 792 & 77 & & 84 & \\
\hline \multicolumn{6}{|l|}{ Smoking } \\
\hline Never & 705 & 87 & \multirow[t]{2}{*}{$<0.001$} & 91 & \multirow[t]{2}{*}{0.002} \\
\hline Ever & 832 & 78 & & 84 & \\
\hline \multicolumn{6}{|l|}{ Tumor size (mm) } \\
\hline$\leq 30$ & 953 & 86 & \multirow[t]{2}{*}{$<0.001$} & 90 & \multirow[t]{2}{*}{$<0.001$} \\
\hline$>30$ & 597 & 75 & & 83 & \\
\hline \multicolumn{6}{|l|}{ Pleural invasion } \\
\hline Absent & 1120 & 88 & \multirow[t]{2}{*}{$<0.001$} & 92 & \multirow[t]{2}{*}{$<0.001$} \\
\hline Present & 430 & 66 & & 75 & \\
\hline \multicolumn{6}{|l|}{ Lymphovascular invasion } \\
\hline Absent & 888 & 92 & \multirow[t]{2}{*}{$<0.001$} & 95 & \multirow[t]{2}{*}{$<0.001$} \\
\hline Present & 662 & 68 & & 76 & \\
\hline \multicolumn{6}{|l|}{ Stage } \\
\hline I & 1112 & 90 & \multirow[t]{4}{*}{$<0.001$} & 94 & \multirow[t]{4}{*}{$<0.001$} \\
\hline II & 245 & 71 & & 78 & \\
\hline III & 187 & 47 & & 57 & \\
\hline \multirow[t]{4}{*}{ IV } & 6 & 80 & & 80 & \\
\hline & \multicolumn{5}{|c|}{ Univariate analysis (Cox model) } \\
\hline & \multicolumn{3}{|l|}{ OS } & \multicolumn{2}{|l|}{ DSS } \\
\hline & HR $\left(95^{c}\right.$ & & $P$ value & HR $(95 \% \mathrm{CI})$ & $P$ value \\
\hline \multicolumn{6}{|l|}{ Histological subtypes } \\
\hline Lepidic vs. Adenocarcinomas with H-FLAC & $0.084(0$ & $.162)$ & $<0.001$ & $0.044(0.018-0.106)$ & $<0.001$ \\
\hline Acinar vs. Adenocarcinomas with H-FLAC & $0.255(0$ & $.453)$ & $<0.001$ & $0.231(0.117-0.458)$ & $<0.001$ \\
\hline Papillary vs. Adenocarcinomas with H-FLAC & $0.316(0$ & $.564)$ & $<0.001$ & $0.261(0.130-0.524)$ & $<0.001$ \\
\hline Solid vs. Adenocarcinomas with H-FLAC & $0.444(0$ & $.799)$ & 0.007 & $0.450(0.225-0.898)$ & 0.023 \\
\hline Micropapillary vs. Adenocarcinomas with H-FLAC & $0.784(0$ & $1.696)$ & 0.536 & $0.798(0.324-1.967)$ & 0.625 \\
\hline Invasive mucinous vs. Adenocarcinomas with H-FLAC & $0.193(0$ & $.426)$ & $<0.001$ & $0.095(0.030-0.303)$ & $<0.001$ \\
\hline
\end{tabular}

$O S$ overall survival, DSS disease-specific survival, $H R$ hazard ratio, $C I$ confidence interval, $H$-FLAC high-grade fetal adenocarcinoma of the lung

specific survival rates of lepidic-, acinar-, papillary-, solid-, and micropapillary-predominant adenocarcinomas, invasive mucinous adenocarcinomas, and adenocarcinomas with the fetal-type component of stage I were 99, 93, 91, 91, 67\%, 97 , and $69 \%$, respectively $(P<0.001)$ (Fig. 3d). When examining only stage I cases, the univariate analysis showed that adenocarcinomas with the fetal-type component had significantly lower overall and disease-specific survival rates than all the other histological subtypes, except for the micropapillary-predominant subtype (Table 8). In the multivariate analysis of stage I cases, the overall survival rate of adenocarcinomas with the fetal-type component was significantly lower than that of all the other histological subtypes except for the micropapillary- and solid-predominant subtypes, and the disease-specific survival rate of adenocarcinomas with the fetal-type component was significantly lower than that of all the other histological subtypes except for the micropapillarypredominant subtype (Table 8). In advanced stage cases, the 5-year overall survival rates of lepidic-, acinar-, papillary-, solid-, and micropapillary-predominant adenocarcinomas, invasive mucinous adenocarcinomas, and adenocarcinomas with the fetal-type component were 84 , $63,62,50,48,69$, and $22 \%$, respectively $(P=0.004)$ (Fig. 3e). The 5-year disease-specific survival rates of lepidic-, acinar-, papillary-, solid-, and micropapillarypredominant adenocarcinomas, invasive mucinous adenocarcinomas, and adenocarcinomas with the fetal-type component of advanced stage were $88,69,75,56,60,88$, and $33 \%$, respectively $(P=0.005)$ (Fig. 3f). Among advanced 

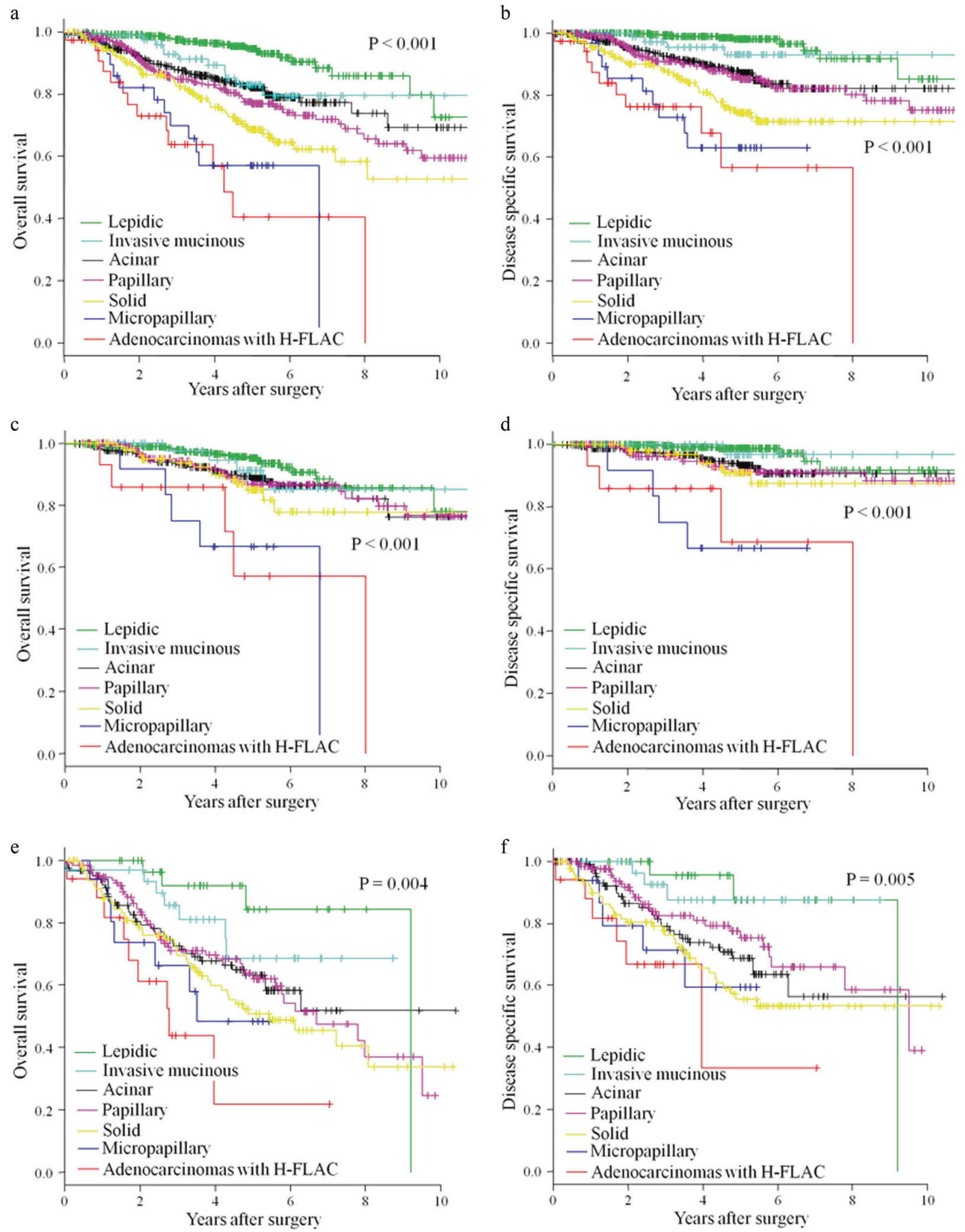

Fig. 3 Kaplan-Meier survival curves of patients with lung adenocarcinomas with high-grade fetal adenocarcinoma (H-FLAC) and major subtypes of lung adenocarcinoma. Overall survival (a) and disease-specific survival (b) curves for histological subgroups in all

stages. Overall survival (c) and disease-specific survival (d) curves for histological subgroups in only stage I cases. Overall survival (e) and disease-specific survival (f) curves for histological subgroups in advanced stage cases 
Table 7 Multivariate analysis for OS and DSS of adenocarcinomas with H-FLAC and major subtypes of lung adenocarcinoma in all stage

\begin{tabular}{|c|c|c|c|c|}
\hline & \multicolumn{2}{|l|}{ OS } & \multicolumn{2}{|l|}{ DSS } \\
\hline & $\mathrm{HR}(95 \% \mathrm{CI})$ & $P$ value & HR $(95 \% \mathrm{CI})$ & $P$ value \\
\hline Age $(>65$ vs. $\leq 65)$ & $1.798(1.386-2.332)$ & $<0.001$ & $1.400(1.018-1.926)$ & 0.038 \\
\hline Smoking (Ever vs. Never) & $1.392(1.067-1.817)$ & 0.015 & $1.198(0.857-1.675)$ & 0.291 \\
\hline Stage (II-IV vs. I) & $3.736(2.865-4.872)$ & $<0.001$ & $4.689(3.320-6.621)$ & $<0.001$ \\
\hline \multicolumn{5}{|l|}{ Histological subtypes } \\
\hline $\begin{array}{l}\text { Lepidic vs. Adenocarcinomas with } \\
\text { H-FLAC }\end{array}$ & $0.174(0.087-0.351)$ & $<0.001$ & $0.094(0.036-0.245)$ & $<0.001$ \\
\hline $\begin{array}{l}\text { Acinar vs. Adenocarcinomas with } \\
\text { H-FLAC }\end{array}$ & $0.424(0.232-0.775)$ & 0.005 & $0.382(0.184-0.794)$ & 0.010 \\
\hline $\begin{array}{l}\text { Papillary vs. Adenocarcinomas with } \\
\text { H-FLAC }\end{array}$ & $0.445(0.243-0.814)$ & 0.009 & $0.346(0.165-0.726)$ & 0.005 \\
\hline $\begin{array}{l}\text { Solid vs. Adenocarcinomas with } \\
\text { H-FLAC }\end{array}$ & $0.535(0.291-0.982)$ & 0.044 & $0.526(0.254-1.089)$ & 0.083 \\
\hline Micropapillary vs. Adenocarcinomas with H-FLAC & $0.890(0.405-1.958)$ & 0.773 & $0.870(0.344-2.202)$ & 0.769 \\
\hline $\begin{array}{l}\text { Invasive mucinous vs. Adenocarcinomas with } \mathrm{H}- \\
\text { FLAC }\end{array}$ & $0.252(0.112-0.568)$ & $<0.001$ & $0.120(0.037-0.394)$ & $<0.001$ \\
\hline
\end{tabular}

OS overall survival, DSS disease-specific survival, $H R$ hazard ratio, $C I$ confidence interval, $H$-FLAC high-grade fetal adenocarcinoma of the lung

stage cases, the univariate analysis showed that adenocarcinomas with the fetal-type component had a significantly lower overall survival rate than all the other histological subtypes except for the micropapillary- and solid-predominant subtypes, and a lower disease-specific survival rate than lepidic- and papillary-predominant adenocarcinomas and invasive mucinous adenocarcinomas (Table 9). The multivariate analysis showed that the overall and disease-specific survival rates of adenocarcinomas with the fetal-type component were significantly lower than that of lepidic-predominant adenocarcinomas and invasive mucinous adenocarcinomas (Table 9).

\section{Discussion}

In this study, the patients with lung cancer with the fetaltype component were predominantly elderly men with a smoking history, and showed admixture with diverse components, such as large cell neuroendocrine carcinoma, squamous cell carcinoma, and enteric adenocarcinoma. These features are similar to findings in past studies [1, 46]. Our past study showed that 6 of 20 lung cancer cases with the fetal-type component were female nonsmokers [6], and the present study of a larger cohort showed that only 3 of 53 cases of lung cancer with the fetal-type component were female nonsmokers, solidifying that high-grade fetal adenocarcinoma is a smoking-associated adenocarcinoma.

In the present study, the fetal-type component was commonly immunopositive for oncofetal markers, including $\alpha$-fetoprotein, glypican-3, and SALL4. Previous studies have also reported that high-grade fetal adenocarcinoma was positive for these markers [1, 4-6], although the expression varied. No one oncofetal marker was positive in over $50 \%$ of the cases, but at least one of the three oncofetal markers was positive in $60 \%$. Immunostaining for these markers may be helpful for distinguishing high-grade fetal adenocarcinoma from conventional lung adenocarcinoma with clear cell features.

As described in previous studies [5, 6], the solid-clear cell pattern was often found to be mixed with the classic glandular pattern of high-grade fetal adenocarcinoma. The solid-clear cell pattern was noted in our original study of the fetal-type component [1]. The current WHO classification of the lung tumors defines high-grade fetal adenocarcinoma as "an adenocarcinoma resembling fetal lung" [2]. Our previous and present studies showed that the solid-clear cell and glandular patterns of the fetal-type component exhibited similar cytomorphology with clear cytoplasm and almost identical immunohistochemical features of the fetal phenotype [6]. Therefore, we believe that the solid-clear cell pattern should be regarded as a poorly differentiated form of high-grade fetal adenocarcinoma rather than a solid pattern of conventional lung adenocarcinoma.

With regard to prognosis, low-grade fetal adenocarcinoma cases are mostly discovered at stage I and have low mortality ( $<10 \%$ of patients) $[1,3,4]$. Meanwhile, this study revealed that the 5-year overall and disease-specific survival rates of adenocarcinomas with the fetal-type component in stage I were 57 and 69\%, respectively, confirming that adenocarcinomas with the fetal-type component have a worse prognosis than low-grade fetal adenocarcinomas. Regarding the prognostic difference between adenocarcinomas with the fetal-type component and conventional adenocarcinomas, both the univariate and multivariate analyses of the present study showed that adenocarcinomas with the fetal-type component had a significantly lower overall survival rate than the other 
Table 8 Univariate and multivariate analyses for OS and DSS of adenocarcinomas with H-FLAC and major subtypes of lung adenocarcinoma in stage I

\begin{tabular}{|c|c|c|c|c|c|}
\hline & \multicolumn{5}{|c|}{ Univariate analysis (log-rank test) } \\
\hline & Number & 5 -yr OS $(\%)$ & $P$ value & 5-yr DSS (\%) & $P$ value \\
\hline \multicolumn{6}{|l|}{ Age (year) } \\
\hline$\leq 65$ & 441 & 94 & $<0.001$ & 96 & 0.245 \\
\hline$>65$ & 671 & 87 & & 93 & \\
\hline \multicolumn{6}{|l|}{ Sex } \\
\hline Female & 577 & 92 & 0.007 & 96 & 0.180 \\
\hline Male & 535 & 87 & & 92 & \\
\hline \multicolumn{6}{|l|}{ Smoking } \\
\hline Never & 537 & 93 & 0.004 & 96 & 0.107 \\
\hline Ever & 565 & 87 & & 93 & \\
\hline \multicolumn{6}{|l|}{ Tumor size $(\mathrm{mm})$} \\
\hline$\leq 30$ & 771 & 92 & $<0.001$ & 95 & 0.002 \\
\hline$>30$ & 341 & 85 & & 91 & \\
\hline \multicolumn{6}{|l|}{ Pleural invasion } \\
\hline Absent & 905 & 92 & $<0.001$ & 96 & $<0.001$ \\
\hline Present & 207 & 78 & & 86 & \\
\hline \multicolumn{6}{|l|}{ Lymphovascular invasion } \\
\hline Absent & 772 & 94 & $<0.001$ & 97 & $<0.001$ \\
\hline \multirow[t]{4}{*}{ Present } & 340 & 81 & & 88 & \\
\hline & & \multicolumn{4}{|c|}{ Univariate analysis (Cox model) } \\
\hline & & \multicolumn{2}{|l|}{ OS } & \multicolumn{2}{|l|}{ DSS } \\
\hline & & $\mathrm{HR}(95 \% \mathrm{CI})$ & $P$ value & $\mathrm{HR}(95 \% \mathrm{CI})$ & $P$ value \\
\hline \multicolumn{6}{|l|}{ Histological subtypes } \\
\hline Lepidic vs Adenocarcinomas with H-FLAC & & $0.123(0.047-0.326)$ & $<0.001$ & $0.048(0.014-0.164)$ & $<0.001$ \\
\hline Acinar vs Adenocarcinomas with H-FLAC & & $0.245(0.096-0.629)$ & 0.003 & $0.166(0.056-0.492)$ & 0.001 \\
\hline Papillary vs Adenocarcinomas with H-FLAC & & $0.245(0.093-0.649)$ & 0.004 & $0.192(0.063-0.587)$ & 0.003 \\
\hline Solid vs Adenocarcinomas with H-FLAC & & $0.308(0.111-0.855)$ & 0.024 & $0.216(0.065-0.718)$ & 0.012 \\
\hline Micropapillary vs Adenocarcinomas with H-FLAC & & $1.031(0.298-3.565)$ & 0.961 & $0.996(0.249-3.990)$ & 0.995 \\
\hline \multirow[t]{4}{*}{ Invasive mucinous vs Adenocarcinomas with H-FLAC } & & $0.165(0.044-0.618)$ & 0.007 & $0.049(0.005-0.440)$ & 0.007 \\
\hline & & \multicolumn{4}{|c|}{ Multivariate analysis (Cox model) } \\
\hline & & \multicolumn{2}{|l|}{ OS } & \multicolumn{2}{|l|}{ DSS } \\
\hline & & $\mathrm{HR}(95 \% \mathrm{CI})$ & $P$ value & $\mathrm{HR}(95 \% \mathrm{CI})$ & $P$ value \\
\hline Age (>65 vs. $\leq 65)$ & & $2.591(1.669-4.021)$ & $<0.001$ & $1.544(0.883-2.700)$ & 0.127 \\
\hline Smoking (Ever vs. Never) & & $1.637(1.087-2.465)$ & 0.018 & $1.311(0.750-2.292)$ & 0.341 \\
\hline \multicolumn{6}{|l|}{ Histological subtypes } \\
\hline Lepidic vs. Adenocarcinomas with H-FLAC & & $0.135(0.050-0.363)$ & $<0.001$ & $0.046(0.013-0.165)$ & $<0.001$ \\
\hline Acinar vs. Adenocarcinomas with H-FLAC & & $0.294(0.114-0.757)$ & 0.011 & $0.186(0.062-0.556)$ & 0.003 \\
\hline Papillary vs. Adenocarcinomas with H-FLAC & & $0.303(0.114-0.805)$ & 0.017 & $0.209(0.067-0.648)$ & 0.007 \\
\hline Solid vs. Adenocarcinomas with H-FLAC & & $0.369(0.132-1.027)$ & 0.056 & $0.239(0.072-0.798)$ & 0.020 \\
\hline Micropapillary vs. Adenocarcinomas with H-FLAC & & $1.443(0.414-5.029)$ & 0.565 & $1.190(0.293-4.831)$ & 0.808 \\
\hline Invasive mucinous vs. Adenocarcinomas with H-FLAC & & $0.177(0.047-0.673)$ & 0.011 & $0.049(0.005-0.453)$ & 0.008 \\
\hline
\end{tabular}

OS overall survival, DSS disease-specific survival, $H R$ hazard ratio, $C I$ confidence interval, $H$-FLAC high-grade fetal adenocarcinoma of the lung

histological subtypes, except for the micropapillarypredominant subtype. In both the univariate and multivariate analyses of stage I cases, adenocarcinomas with the fetal-type component had a significantly lower diseasespecific survival rate than the other histological subtypes, except for the micropapillary-predominant subtype. Past studies of major histological subtypes of lung adenocarcinomas demonstrated that lung adenocarcinomas with solid and micropapillary predominant subtypes belong to the poor prognostic group [11-14]. Adenocarcinoma with the fetal-type component is thought to have a poor prognosis, comparable to that of micropapillary adenocarcinoma. The prognostic analysis of 41 cases of adenocarcinoma with the fetal-type component revealed that there 
Table 9 Univariate and multivariate analyses for OS and DSS of adenocarcinomas with H-FLAC and major subtypes of lung adenocarcinoma in advanced stage cases

\begin{tabular}{|c|c|c|c|c|c|}
\hline & \multicolumn{5}{|c|}{ Univariate analysis (log-rank test) } \\
\hline & Number & $5-\mathrm{yr}$ OS $(\%)$ & $P$ value & 5 -yr DSS (\%) & $P$ value \\
\hline \multicolumn{6}{|l|}{ Age (year) } \\
\hline$\leq 65$ & 185 & 63 & 0.072 & 69 & 0.317 \\
\hline$>65$ & 253 & 58 & & 69 & \\
\hline \multicolumn{6}{|l|}{ Sex } \\
\hline Female & 181 & 66 & 0.020 & 76 & 0.052 \\
\hline Male & 257 & 56 & & 64 & \\
\hline \multicolumn{6}{|l|}{ Smoking } \\
\hline Never & 168 & 66 & 0.024 & 75 & 0.082 \\
\hline Ever & 267 & 56 & & 65 & \\
\hline \multicolumn{6}{|l|}{ Tumor size $(\mathrm{mm})$} \\
\hline$\leq 30$ & 182 & 59 & 0.714 & 67 & 0.634 \\
\hline$>30$ & 256 & 61 & & 70 & \\
\hline \multicolumn{6}{|l|}{ Pleural invasion } \\
\hline Absent & 215 & 66 & 0.008 & 73 & 0.050 \\
\hline Present & 223 & 54 & & 65 & \\
\hline \multicolumn{6}{|l|}{ Lymphovascular invasion } \\
\hline Absent & 116 & 76 & 0.003 & 86 & $<0.001$ \\
\hline \multirow[t]{4}{*}{ Present } & 322 & 54 & & 63 & \\
\hline & & \multicolumn{4}{|c|}{ Univariate analysis (Cox model) } \\
\hline & & \multicolumn{2}{|l|}{ OS } & \multicolumn{2}{|l|}{ DSS } \\
\hline & & HR $(95 \% \mathrm{CI})$ & $P$ value & $\mathrm{HR}(95 \% \mathrm{CI})$ & $P$ value \\
\hline \multicolumn{6}{|l|}{ Histological subtypes } \\
\hline \multicolumn{2}{|l|}{ Lepidic vs. Adenocarcinomas with H-FLAC } & $0.126(0.039-0.409)$ & $<0.001$ & $0.137(0.034-0.549)$ & 0.005 \\
\hline \multicolumn{2}{|l|}{ Acinar vs. Adenocarcinomas with H-FLAC } & $0.406(0.196-0.843)$ & 0.016 & $0.443(0.183-1.074)$ & 0.072 \\
\hline \multicolumn{2}{|l|}{ Papillary vs. Adenocarcinomas with H-FLAC } & $0.416(0.202-0.854)$ & 0.017 & $0.349(0.143-0.853)$ & 0.021 \\
\hline \multicolumn{2}{|l|}{ Solid vs. Adenocarcinomas with H-FLAC } & $0.515(0.249-1.062)$ & 0.072 & $0.590(0.246-1.417)$ & 0.238 \\
\hline \multicolumn{2}{|c|}{ Micropapillary vs. Adenocarcinomas with H-FLAC } & $0.627(0.233-1.688)$ & 0.356 & $0.649(0.197-2.131)$ & 0.476 \\
\hline \multicolumn{2}{|c|}{ Invasive mucinous vs. Adenocarcinomas with H-FLAC } & $0.239(0.089-0.642)$ & 0.005 & $0.148(0.037-0.595)$ & 0.007 \\
\hline & & \multicolumn{4}{|c|}{ Multivariate analysis (Cox model) } \\
\hline & & \multicolumn{2}{|l|}{ OS } & \multicolumn{2}{|l|}{ DSS } \\
\hline & & HR $(95 \% \mathrm{CI})$ & $P$ value & $\mathrm{HR}(95 \% \mathrm{CI})$ & $P$ value \\
\hline Age (>65 vs. $\leq 65$ ) & & $1.021(1.005-1.038)$ & 0.012 & $1.017(0.998-1.038)$ & 0.085 \\
\hline Smoking (Ever vs. Never) & & $1.279(0.901-1.815)$ & 0.168 & $1.193(0.785-1.813)$ & 0.409 \\
\hline \multicolumn{6}{|l|}{ Histological subtypes } \\
\hline \multicolumn{2}{|l|}{ Lepidic vs. Adenocarcinomas with H-FLAC } & $0.158(0.047-0.535)$ & 0.003 & $0.178(0.042-0.765)$ & 0.020 \\
\hline \multicolumn{2}{|l|}{ Acinar vs. Adenocarcinomas with H-FLAC } & $0.505(0.232-1.099)$ & 0.085 & $0.573(0.217-1.509)$ & 0.260 \\
\hline \multicolumn{2}{|l|}{ Papillary vs. Adenocarcinomas with H-FLAC } & $0.532(0.246-1.149)$ & 0.108 & $0.461(0.173-1.230)$ & 0.122 \\
\hline Solid vs. Adenocarcinomas with H-FLAC & & $0.643(0.299-1.381)$ & 0.258 & $0.772(0.298-1.997)$ & 0.593 \\
\hline Micropapillary vs. Adenocarcinomas with $\mathrm{H}$ & [-FLAC & $0.742(0.269-2.053)$ & 0.567 & $0.810(0.234-2.809)$ & 0.740 \\
\hline Invasive mucinous vs. Adenocarcinomas witl & h H-FLAC & $0.281(0.101-0.787)$ & 0.016 & $0.182(0.043-0.774)$ & 0.021 \\
\hline
\end{tabular}

$O S$ overall survival, DSS disease-specific survival, $H R$ hazard ratio, $C I$ confidence interval, $H$-FLAC high-grade fetal adenocarcinoma of the lung

was no significant difference in survival between the fetaltype-predominant and fetal-type-nonpredominant subgroups, and that prognosis did not correlate with whether tumors included a solid/micropapillary component. These results indicate that the presence of the fetal-type component, regardless of its proportion (if at least 5\%), is an independent poor prognostic factor in lung adenocarcinomas. This is similar to the prognostic relevance of nonpredominant solid and micropapillary patterns in lung adenocarcinomas [15-18]. The current WHO classification of lung tumors describes that high-grade fetal adenocarcinoma is rendered as a diagnosis when this component is predominant [2]. The results of the present study indicate that pathologists should report the presence of the fetal-type component even when it is nonpredominant, as it may drastically affect survival. 
High-grade fetal adenocarcinoma is thought to be a characteristic lung tumor having a fetal lung-like morphology with the expression of oncofetal markers. Our previous study [6] revealed that the fetal-type component frequently showed immunoexpression of neuroendocrine (chromogranin A, synaptophysin, and CD56) and proneural (BRN2 and ASCL1) markers, indicating the close association of neuroendocrine/neuronal differentiation and high-grade fetal adenocarcinoma. The fetal-type component was also reported to express an intestinal marker, CDX2 [6]. These features appear to recapitulate the developmental state of fetal lung epithelium or endodermal development [19-21]. Carcinomas with primitive signatures have been known to occur in a variety of organs. It has been reported that gastric adenocarcinomas with enteroblastic differentiation frequently express $\alpha$-fetoprotein, glypican-3, and SALL-4 [22-27]. They also display characteristic histology of fetal gut-like morphology, consisting of columnar clear cells [22-27]. These features are very similar to those of high-grade fetal adenocarcinoma of the lung, suggesting they are parallel tumors of different organs with a primitive phenotype, as we stated in our earlier studies [1, 6]. Both tumors represent aggressive biological behavior [26, 27], but it is unknown what kind of molecular mechanism contributes to the primitive phenotype and high-grade malignant nature. Previous studies reported lower frequencies of major driver mutations, such as EGFR and $K R A S$, in lung cancers with the fetal-type component compared to those in common lung adenocarcinomas in East Asian patients $[5,6]$. It is speculated that these tumors may harbor a common specific molecular background. Further studies are indicated to identify the molecular oncogenic mechanisms associated with the fetal phenotype and aggressive nature of these tumors. A key molecular disorder related to the fetal phenotype may possibly cause coexistence with various histological components.

In this study, we evaluated lung cancer stage according to the previous $7^{\text {th }}$ edition of the UICC TNM classification and performed prognostic analyses. The new 8th edition of the UICC TNM classification published in December 2016 evaluates the $\mathrm{T}$ factor according to the invasive size, not the total tumor size [8]. An additional study may be needed to confirm whether prognostic data similar to that of the present study may also be obtained under the new TNM classification.

In conclusion, the high-grade fetal adenocarcinoma component often shows immunoexpression of oncofetal markers and is not uncommonly combined with diverse histological components. Our study demonstrated that lung adenocarcinomas with the fetal-type component had a poor prognosis that was comparable to that of micropapillary adenocarcinoma. The presence of the high-grade fetal adenocarcinoma component in lung adenocarcinomas should be recognized as an important prognostic marker.
Acknowledgements We are grateful to the technical staff in the Department of Pathology, Kanagawa Cancer Center for their expert technical assistance.

\section{Compliance with ethical standards}

Conflict of interest The authors declare that they have no conflict of interest.

\section{References}

1. Nakatani Y, Kitamura H, Inayama Y, et al. Pulmonary adenocarcinomas of the fetal lung type: a clinicopathologic study indicating differences in histology, epidemiology, and natural history of low-grade and high-grade forms. Am J Surg Pathol. 1998;22:399-411.

2. Travis WD, Brambilla E, Burke AP, et al. WHO classification of tumours of the lung, pleura, thymus and heart. Lyon: IARC Press; 2015.

3. Sato S, Koike T, Yamato Y, et al. Resected well-differentiated fetal pulmonary adenocarcinoma and summary of 25 cases reported in Japan. Jpn J Thorac Cardiovasc Surg. 2006;54:539-42.

4. Zhang J, Sun J, Liang XL, et al. Differences between low and high grade fetal adenocarcinoma of the lung: a clinicopathological and molecular study. J Thorac Dis. 2017;9:2071-8.

5. Morita S, Yoshida A, Goto A, et al. High-grade lung adenocarcinoma with fetal lung-like morphology: clinicopathologic, immunohistochemical, and molecular analyses of 17 cases. Am J Surg Pathol. 2013;37:924-32.

6. Suzuki M, Yazawa T, Ota S, et al. High-grade fetal adenocarcinoma of the lung is a tumour with a fetal phenotype that shows diverse differentiation, including high-grade neuroendocrine carcinoma: a clinicopathological, immunohistochemical and mutational study of 20 cases. Histopathology. 2015;67:806-16.

7. Travis WD, Brambilla E, Noguchi M, et al. International Association for the Study of Lung Cancer/American Thoracic Society/ European Respiratory Society International Multidisciplinary Classification of Lung Adenocarcinoma. J Thorac Oncol. 2011;6: 244-85.

8. James DB, Mary KG, Christian W (editors). TNM classification of malignant tumors. 8th ed. Hoboken: Wiley-Blackwell; 2016.

9. Leslie HS, Mary KG, Christian W. TNM classification of malignant tumors. 7th ed. Hoboken: Wiley-Blackwell; 2009.

10. Kanda Y. Investigation of the freely available easy-to-use software 'EZR' for medical statistics. Bone Marrow Transplant. 2013;48:452-8.

11. Yoshizawa A, Motoi N, Riely GJ, et al. Impact of proposed IASLC/ATS/ERS classification of lung adenocarcinoma: prognostic subgroups and implications for further revision of staging based on analysis of 514 stage I cases. Mod Pathol. 2011;24: 653-64.

12. Warth A, Muley T, Meister M, et al. The novel histologic International Association for the Study of Lung Cancer/American Thoracic Society/European Respiratory Society classification system of lung adenocarcinoma is a stage-independent predictor of survival. J Clin Oncol. 2012;30:1438-46.

13. Tsuta K, Kawago M, Inoue E, et al. The utility of the proposed IASLC/ATS/ERS lung adenocarcinoma subtypes for disease prognosis and correlation of driver gene alterations. Lung Cancer. 2013;81:371-6.

14. Yoshizawa A, Sumiyoshi S, Sonobe M, et al. Validation of the IASLC/ATS/ERS lung adenocarcinoma classification for prognosis and association with EGFR and KRAS gene mutations: analysis of 440 Japanese patients. J Thorac Oncol. 2013;8:52-61. 
15. Suzuki M, Yokose T, Nakayama H. Prognostic contribution of non-predominant solid and micropapillary components in lung adenocarcinomas. J Thorac Dis. 2017;9:504-6.

16. Kamiya K, Hayashi Y, Douguchi J, et al. Histopathological features and prognostic significance of the micropapillary pattern in lung adenocarcinoma. Mod Pathol. 2008;21:992-1001.

17. Zhao Y, Wang R, Shen X, et al. Minor components of micropapillary and solid subtypes in lung adenocarcinoma are predictors of lymph node metastasis and poor prognosis. Ann Surg Oncol. 2016;23:2099-105.

18. Yanagawa N, Shiono S, Abiko M, et al. The clinical impact of solid and micropapillary patterns in resected lung adenocarcinoma. J Thorac Oncol. 2016;11:1976-83.

19. Nakatani Y. Pulmonary endocrine cells in infancy and childhood. Pediatr Pathol. 1991;11:31-48.

20. Volante M, Fulcheri E, Allia E, et al. Ghrelin expression in fetal, infant, and adult human lung. J Histochem Cytochem. 2002;50:1013-21.

21. Morrisey EE, Hogan BL. Preparing for the first breath: genetic and cellular mechanisms in lung development. Dev Cell. 2010;18:8-23.

22. Matsunou H, Konishi F, Jalal RE, et al. Alpha-fetoproteinproducing gastric carcinoma with enteroblastic differentiation. Cancer. 1994;73:534-40.
23. Hishinuma M, Ohashi KI, Yamauchi N, et al. Hepatocellular oncofetal protein, glypican 3 is a sensitive marker for alphafetoprotein-producing gastric carcinoma. Histopathology. 2006;49:479-86.

24. Ushiku T, Uozaki H, Shinozaki A, et al. Glypican 3-expressing gastric carcinoma: distinct subgroup unifying hepatoid, clear-cell, and $\alpha$-fetoprotein-producing gastric carcinomas. Cancer Sci. 2009;100:626-32.

25. Ushiku T, Shinozaki A, Shibahara J, et al. SALL4 represents fetal gut differentiation of gastric cancer, and is diagnostically useful in distinguishing hepatoid gastric carcinoma from hepatocellular carcinoma. Am J Surg Pathol. 2010;34:533-40.

26. Yamazawa S, Ushiku T, Shinozaki-Ushiku A, et al. Gastric cancer with primitive enterocyte phenotype: an aggressive subgroup of intestinal-type adenocarcinoma. Am J Surg Pathol. 2017;41: 989-97.

27. Murakami T, Yao T, Mitomi H, et al. Clinicopathologic and immunohistochemical characteristics of gastric adenocarcinoma with enteroblastic differentiation: a study of 29 cases. Gastric Cancer. 2016;19:498-507. 